\title{
GENERALIZATIONS OF THE BERNOULLI AND APPELL POLYNOMIALS
}

\author{
GABRIELLA BRETTI, PIERPAOLO NATALINI, AND PAOLO E. RICCI
}

Received 19 July 2002

We first introduce a generalization of the Bernoulli polynomials, and consequently of the Bernoulli numbers, starting from suitable generating functions related to a class of Mittag-Leffler functions. Furthermore, multidimensional extensions of the Bernoulli and Appell polynomials are derived generalizing the relevant generating functions, and using the Hermite-Kampé de Fériet (or Gould-Hopper) polynomials. The main properties of these polynomial sets are shown. In particular, the differential equations can be constructed by means of the factorization method.

\section{Introduction}

A recent paper [7] deals with generalized forms of Bernoulli polynomials, used in order to derive explicit summation formulas, generalizing well-known classical results. Furthermore, the Appell polynomials were applied for the construction of quadrature rules involving Appell instead of Bernoulli polynomials $[4,6]$. In our opinion, the technique introduced in [7] using the Hermite-Kampé de Fériet (or Gould-Hopper) polynomials in order to extend to several variables many classical univariable formulas could be exploited in order to find further generalizations of the above results, permitting the construction of new summation formulas and multidimensional quadrature rules.

A preliminary analysis of the main properties of generalized Bernoulli or Appell polynomials is included in this paper.

\section{Recalling Bernoulli and Appell polynomials}

The Bernoulli polynomials $B_{n}(x)$ can be defined by means of the generating function

$$
G(x, t):=\frac{t e^{x t}}{e^{t}-1}=\sum_{n=0}^{\infty} B_{n}(x) \frac{t^{n}}{n !}, \quad|t|<2 \pi .
$$

By putting $x=0$, we obtain the Bernoulli numbers $B_{n}:=B_{n}(0)$ and the relevant generating function 
614 Generalizations of the Bernoulli and Appell polynomials

$$
\frac{t}{e^{t}-1}=\sum_{n=0}^{\infty} B_{n} \frac{t^{n}}{n !} \text {. }
$$

It is well known that

$$
\begin{aligned}
& B_{n}(0)=B_{n}(1)=B_{n}, \quad n \neq 1, \\
& B_{n}(x)=\sum_{k=0}^{n}\left(\begin{array}{l}
n \\
k
\end{array}\right) B_{k} x^{n-k}, \\
& B_{n}^{\prime}(x)=n B_{n-1}(x) .
\end{aligned}
$$

The Bernoulli numbers enter into many mathematical formulas, such as

(i) the Taylor expansion in a neighborhood of the origin of the circular and hyperbolic tangent and cotangent functions,

(ii) the sums of powers of natural numbers,

(iii) the remainder term of the Euler-MacLaurin quadrature rule.

The Bernoulli polynomials, first studied by Euler, are employed in the integral representation of differentiable periodic functions, since they are employed for approximating such functions in terms of polynomials. They are also used for representing the remainder term of the composite Euler-MacLaurin quadrature rule (see [22]).

The Appell polynomials [2] are defined by the generating function

$$
G_{A}(x, t)=A(t) e^{x t}=\sum_{n=0}^{\infty} \frac{R_{n}(x)}{n !} t^{n}
$$

where

$$
A(t)=\sum_{k=0}^{\infty} \frac{\mathscr{R}_{k}}{k !} t^{k}, \quad A(0) \neq 0,
$$

is an analytic function at $t=0$, and $\mathscr{R}_{k}:=R_{k}(0)$.

It is easy to see that for any $A(t)$, the derivatives of $R_{n}(x)$ satisfy

$$
R_{n}^{\prime}(x)=n R_{n-1}(x)
$$

and furthermore,

(i) if $A(t)=t /\left(e^{t}-1\right)$, then $R_{n}(x)=B_{n}(x)$,

(ii) if $A(t)=2 /\left(e^{t}+1\right)$, then $R_{n}(x)=E_{n}(x)$, the Euler polynomials,

(iii) if $A(t)=\alpha_{1} \cdots \alpha_{m} t^{m}\left[\left(e^{\alpha_{1} t}-1\right) \cdots\left(e^{\alpha_{m} t}-1\right)\right]^{-1}$, then the $R_{n}(x)$ are the Bernoulli polynomials of order $m$ (see [11]),

(iv) if $A(t)=2^{m}\left[\left(e^{\alpha_{1} t}+1\right) \cdots\left(e^{\alpha_{m} t}+1\right)\right]^{-1}$, then the $R_{n}(x)$ are the Euler polynomials of order $m$ (see [11]),

(v) if $A(t)=e^{\xi_{0}+\xi_{1} t+\cdots+\xi_{d+1} t^{d+1}}, \xi_{d+1} \neq 0$, then the $R_{n}(x)$ are the generalized GouldHopper polynomials (see [10]), including the Hermite polynomials when $d=1$ and classical 2-orthogonal polynomials when $d=2$. 


\section{Generalizations of the Bernoulli polynomials}

Some generalized forms of the Bernoulli polynomials and numbers already appeared in literature:

(i) the generalized Bernoulli polynomials $B_{n}^{\alpha}(x)$ defined by the generating function

$$
\frac{t^{\alpha} e^{x t}}{\left(e^{t}-1\right)^{\alpha}}=\sum_{n=0}^{\infty} B_{n}^{\alpha}(x) \frac{t^{n}}{n !}, \quad|t|<2 \pi
$$

by means of which Tricomi and Erdélyi [23] gave an asymptotic expansion of the ratio of gamma functions;

(ii) the polynomials of Nath [19], defined by the generating function

$$
\frac{(h t)^{\alpha}(1+w t)^{x / w}}{\left[(1+w t)^{h / w}-1\right]^{\alpha}}=\sum_{n=0}^{\infty} B_{n ; h, w}^{\alpha}(x) \frac{t^{n}}{n !}, \quad|t|<\left|\frac{1}{w}\right|
$$

(iii) the polynomials of Frappier [12], defined by the generating function

$$
\frac{(i z)^{\alpha} e^{(x-1 / 2) z}}{2^{2 \alpha} \Gamma(\alpha+1) J_{\alpha}(i z / 2)}=\sum_{n=0}^{\infty} B_{n, \alpha}(x) \frac{z^{n}}{n !}, \quad|z|<2\left|j_{1}\right|,
$$

where $J_{\alpha}$ is the Bessel function of the first kind of order $\alpha$ and $j_{1}=j_{1}(\alpha)$ is the first zero of $J_{\alpha}$.

\section{A new class of generalized Bernoulli polynomials: $B_{n}^{[m-1]}(x), m \geq 1$}

In this section, we introduce a countable set of polynomials $B_{n}^{[m-1]}(x)$ generalizing the Bernoulli ones (which can be recovered assuming that $m=1$ ), introduced by Natalini and Bernardini [18].

To this end, we consider a class of Appell polynomials, defined by using a generating function linked to the so-called Mittag-Leffler function,

$$
E_{1, m+1}(t):=\frac{t^{m}}{e^{t}-\sum_{h=0}^{m-1}\left(t^{h} / h !\right)}
$$

considered in the general form by Agarwal [1].

The generalized Bernoulli polynomials $B_{n}^{[m-1]}(x), m \geq 1$, are defined by the generating function

$$
G^{[m-1]}(x, t):=\frac{t^{m} e^{x t}}{e^{t}-\sum_{h=0}^{m-1}\left(t^{h} / h !\right)}=\sum_{n=0}^{\infty} B_{n}^{[m-1]}(x) \frac{t^{n}}{n !} .
$$


616 Generalizations of the Bernoulli and Appell polynomials

For $m=1$, we obtain, from the above equation, the generating function $G^{(0)}(x, t)=$ $t e^{x t} /\left(e^{t}-1\right)$ of the classical Bernoulli polynomials $B_{n}^{(0)}(x)$.

Since $G^{[m-1]}(x, t)=A(t) e^{x t}$, the generalized Bernoulli polynomial belong to the class of Appell polynomials.

It is possible to define the generalized Bernoulli numbers assuming that

$$
B_{n}^{[m-1]}=B_{n}^{[m-1]}(0)
$$

The following properties are proved in the above-mentioned paper [18].

(i) Explicit forms:

$$
x^{n}=\sum_{h=0}^{n}\left(\begin{array}{l}
n \\
h
\end{array}\right) \frac{h !}{(h+m) !} B_{n-h}^{[m-1]}(x) .
$$

Inverting this equation, it is possible to find explicit expressions for the polynomials $B_{n}^{[m-1]}(x)$. The first ones are given by

$$
\begin{gathered}
B_{0}^{[m-1]}(x)=m ! \\
B_{1}^{[m-1]}(x)=m !\left(x-\frac{1}{m+1}\right), \\
B_{2}^{[m-1]}(x)=m !\left(x^{2}-\frac{2}{m+1} x+\frac{2}{(m+1)^{2}(m+2)}\right),
\end{gathered}
$$

and, consequently, the first generalized Bernoulli numbers are

$$
\begin{gathered}
B_{0}^{[m-1]}=m !, \quad B_{1}^{[m-1]}=-\frac{m !}{m+1}, \\
B_{2}^{[m-1]}=\frac{2 m !}{(m+1)^{2}(m+2)} .
\end{gathered}
$$

(ii) Recurrence relation for the $B_{n}^{[m-1]}$ polynomials:

$$
B_{n}^{[m-1]}(x)=\left(x-\frac{1}{m+1}\right) B_{n-1}^{[m-1]}(x)-\frac{1}{n(m-1) !} \sum_{k=0}^{n-2}\left(\begin{array}{l}
n \\
k
\end{array}\right) B_{n-k}^{[m-1]} B_{k}^{[m-1]}(x) .
$$

This relation, starting from $n=1$, and taking into account the initial value $B_{0}^{[m-1]}(x)=$ $m$ !, allows a recursive computation for this class of generalized Bernoulli polynomials.

(iii) Differential equation for the $B_{n}^{[m-1]}$ polynomials:

$$
\begin{aligned}
& \frac{B_{n}^{[m-1]}}{n !} y^{(n)}+\frac{B_{n-1}^{[m-1]}}{(n-1) !} y^{(n-1)}+\cdots+\frac{B_{2}^{[m-1]}}{2 !} y^{\prime \prime} \\
& \quad+(m-1) !\left(\frac{1}{m+1}-x\right) y^{\prime}+n(m-1) ! y=0 .
\end{aligned}
$$


This is an equation of order $n$ so that all the considered families of polynomials can be viewed as solutions of differential operators of infinite order.

Remark 4.1. Note that the generating function could be written in the form

$$
G^{[m-1]}(x, t):=\frac{t^{m} e^{x t}}{e^{t}-\sum_{h=0}^{m-1}\left(t^{h} / h !\right)}=m ! \sum_{n=0}^{\infty} \tilde{B}_{n}^{[m-1]}(x) \frac{t^{n}}{n !}
$$

so that, putting

$$
B_{n}^{[m-1]}(x)=m ! \tilde{B}_{n}^{[m-1]}(x),
$$

we obtain the explicit form of the generalized Bernoulli polynomial $\tilde{B}_{n}^{[m-1]}$ from the preceding one simply by dividing by $m$ !, and so decreasing the relevant numerical values.

\section{2D extensions of the Bernoulli and Appell polynomials}

The Hermite-Kampé de Fériet [3] (or Gould-Hopper) polynomials [13, 21] have been used recently in order to construct addition formulas for different classes of generalized Gegenbauer polynomials [9].

They are defined by the generating function

$$
e^{x t+y t^{j}}=\sum_{n=0}^{\infty} H_{n}^{(j)}(x, y) \frac{t^{n}}{n !}
$$

or by the explicit form

$$
H_{n}^{(j)}(x, y)=n ! \sum_{s=0}^{[n / j]} \frac{x^{n-j s} y^{s}}{(n-j s) ! s !}
$$

where $j \geq 2$ is an integer. The case when $j=1$ is not considered since the corresponding $2 \mathrm{D}$ polynomials are simply expressed by the Newton binomial formula.

It is worth recalling that the polynomials $H_{n}^{(j)}(x, y)$ are a natural solution of the generalized heat equation

$$
\frac{\partial}{\partial y} F(x, y)=\frac{\partial^{j}}{\partial x^{j}} F(x, y), \quad F(x, 0)=x^{n} .
$$

The case when $j=2$ is then particularly important (see Widder [24]); it was recently used in order to define 2D extensions of the Bernoulli and Euler polynomials [7].

Further generalizations including the $H_{n}^{(j)}(x, y)$ polynomials as a particular case are given by

$$
e^{x_{1} t+x_{2} t^{2}+\cdots+x_{r} t^{r}}=\sum_{n=0}^{\infty} H_{n}\left(x_{1}, x_{2}, \ldots, x_{r}\right) \frac{t^{n}}{n !} .
$$


Note that the generating function of the last equation can be written in the form

$$
\begin{aligned}
e^{x_{1} t+x_{2} t^{2}+\cdots+x_{r} t^{r}} & =\sum_{k=0}^{\infty} \frac{\left(x_{1} t+x_{2} t^{2}+\cdots+x_{r} t^{r}\right)^{k}}{k !} \\
& =\sum_{k=0}^{\infty} \frac{1}{k !} \sum_{k_{1}+k_{2}+\cdots+k_{r}=k} \frac{k !}{k_{1} ! k_{2} ! \cdots k_{r} !} x_{1}^{k_{1}} x_{2}^{k_{2}} \cdots x_{r}^{k_{r}} t^{k_{1}+2 k_{2}+\cdots+r k_{r}} \\
& =\sum_{n=0}^{\infty}\left(\sum_{\pi_{k}(n \mid r)} n ! \frac{x_{1}^{k_{1}} x_{2}^{k_{2}} \cdots x_{r}^{k_{r}}}{k_{1} ! k_{2} ! \cdots k_{r} !}\right) \frac{t^{n}}{n !},
\end{aligned}
$$

where $k:=k_{1}+k_{2}+\cdots+k_{r}, n:=k_{1}+2 k_{2}+\cdots+r k_{r}$, and the sum runs over all the restricted partitions $\pi_{k}(n \mid r)$ (containing at most $r$ sizes) of the integer $n$, with $k$ denoting the number of parts of the partition and $k_{i}$ the number of parts of size $i$. Note that, using the ordinary notation for the partitions of $n$, that is, $n=k_{1}+2 k_{2}+\cdots+n k_{n}$, we have to assume $k_{r+1}=k_{r+2}=\cdots=k_{n}=0$.

Consequently, the explicit form of the multidimensional Hermite-Kampé de Fériet polynomials

$$
H_{n}\left(x_{1}, x_{2}, \ldots, x_{r}\right)=\sum_{\pi_{k}(n \mid r)} n ! \frac{x_{1}^{k_{1}} x_{2}^{k_{2}} \cdots x_{r}^{k_{r}}}{k_{1} ! k_{2} ! \cdots k_{r} !}
$$

follows.

Furthermore, they satisfy for every $n$ the isobaric property (of weight $n$ )

$$
H_{n}\left(t x_{1}, t^{2} x_{2}, \ldots, t^{r} x_{r}\right)=t^{n} H_{n}\left(x_{1}, x_{2}, \ldots, x_{r}\right),
$$

and consequently, they are solutions of the first-order partial differential equation

$$
x_{1} \frac{\partial H_{n}}{\partial x_{1}}+2 x_{2} \frac{\partial H_{n}}{\partial x_{2}}+\cdots+r x_{r} \frac{\partial H_{n}}{\partial x_{r}}=n H_{n}
$$

The multivariate Hermite-Kampé de Fériet polynomials appear as an interesting tool for introducing and studying multidimensional generalizations of the Appell polynomials too, including the Bernoulli and Euler ones, starting from the corresponding generating functions. A first approach in this direction was given in [8].

In the following, we recall some results of Bretti and Ricci [5], presenting some properties of the generalized 2D Appell polynomials, but considering first the case of the 2D Bernoulli polynomials, in order to introduce the subject in a more friendly way. The relevant extensions to the multidimensional Bernoulli and Appell case can be derived almost straightforwardly, but the relevant equations are rather involved.

We will show that for every integer $j \geq 2$, it is possible to define a class of 2D Bernoulli polynomials denoted by $B_{n}^{(j)}(x, y)$ generalizing the classical Bernoulli polynomials. 
Furthermore, the bivariate Appell polynomials $R_{n}^{(j)}(x, y)$ are introduced by means of the generating function

$$
A(t) e^{x t+y t^{j}}=\sum_{n=0}^{\infty} R_{n}^{(j)}(x, y) \frac{t^{n}}{n !} .
$$

Exploiting the factorization method (see $[14,16]$ ), we show how to derive the differential equations satisfied by these $2 \mathrm{D}$ polynomials. The differential equation for the classical Appell polynomials was first obtained by Sheffer [20], and was recently recovered in [15].

Remark 5.1. It is worth noting that recently Professor Ismail [17], avoiding the use of the factorization method, was able to prove that the differential equation of infinite order satisfied by the Appell polynomials is nothing special since it can be stated for a general polynomial family.

Further generalizations are given by the multiindex polynomials defined by means of the generating functions

$$
A(t, \tau) e^{x t^{l}+y \tau^{j}}=\sum_{n, m=0}^{\infty} R_{n, m}^{(l, j)}(x, y) \frac{t^{n}}{n !} \frac{\tau^{m}}{m !}
$$

or, more generally,

$$
A\left(t_{1}, \ldots, t_{r}\right) e^{x_{1} t_{1}^{j_{1}}+\cdots+x_{r} t_{r}^{j_{r}}}=\sum_{n_{1}, \ldots, n_{r}=0}^{\infty} R_{n_{1}, \ldots, n_{r}}^{\left(j_{1}, \ldots, j_{r}\right)}\left(x_{1}, \ldots, x_{r}\right) \frac{t_{1}^{n_{1}}}{n_{1} !} \cdots \frac{t_{r}^{n_{r}}}{n_{r} !},
$$

which belong to the set of multidimensional special functions recently introduced by Dattoli and his group.

\section{The 2D Bernoulli polynomials $B_{n}^{(j)}(x, y)$}

Starting from the Hermite-Kampé de Fériet (or Gould-Hopper) polynomials $H_{n}^{(j)}(x, y)$, we define the 2D Bernoulli polynomials $B_{n}^{(j)}(x, y)$ by means of the generating function

$$
G^{(j)}(x, y ; t):=\frac{t}{e^{t}-1} e^{x t+y t^{j}}=\sum_{n=0}^{\infty} B_{n}^{(j)}(x, y) \frac{t^{n}}{n !} .
$$

It is worth noting that the polynomial $H_{n}^{(j)}(x, y)$, being isobaric of weight $n$, cannot contain the variable $y$, for every $n=0,1, \ldots, j-1$.

The following results for the $B_{n}^{(j)}(x, y)$ polynomials can be derived.

(i) Explicit forms of the polynomials $B_{n}^{(j)}$ in terms of the Hermite-Kampé de Fériet polynomials $H_{n}^{(j)}$ and vice versa:

$$
B_{n}^{(j)}(x, y)=\sum_{h=0}^{n}\left(\begin{array}{l}
n \\
h
\end{array}\right) B_{n-h} H_{n}^{(j)}(x, y)=n ! \sum_{h=0}^{n} \frac{B_{n-h}}{(n-h) !} \sum_{r=0}^{[h / j]} \frac{x^{h-j r} y^{r}}{(h-j r) ! r !},
$$


where $B_{k}$ denote the Bernoulli numbers;

$$
H_{n}^{(j)}(x, y)=\sum_{h=0}^{n}\left(\begin{array}{l}
n \\
h
\end{array}\right) \frac{1}{n-h+1} B_{h}^{(j)}(x, y) .
$$

(ii) Recurrence relation:

$$
\begin{aligned}
B_{0}^{(j)}(x, y)=1 \\
B_{n}^{(j)}(x, y)=-\frac{1}{n} \sum_{k=0}^{n-2}\left(\begin{array}{l}
n \\
k
\end{array}\right) B_{n-k} B_{k}^{(j)}(x, y) \\
+\left(x-\frac{1}{2}\right) B_{n-1}^{(j)}(x, y)+j y \frac{(n-1) !}{(n-j) !} B_{n-j}^{(j)}(x, y) .
\end{aligned}
$$

(iii) Shift operators:

$$
\begin{gathered}
L_{n}^{-}:=\frac{1}{n} D_{x}, \quad L_{n}^{+}:=\left(x-\frac{1}{2}\right)-\sum_{k=0}^{n-1} \frac{B_{n-k+1}}{(n-k+1) !} D_{x}^{n-k}+j y D_{x}^{j-1}, \\
\mathscr{L}_{n}^{-}:=\frac{1}{n} D_{x}^{-(j-1)} D_{y}, \\
\mathscr{L}_{n}^{+}:=\left(x-\frac{1}{2}\right)+j y D_{x}^{-(j-1)^{2}} D_{y}^{j-1}-\sum_{k=0}^{n-1} \frac{B_{n-k+1}}{(n-k+1) !} D_{x}^{-(j-1)(n-k)} D_{y}^{n-k} .
\end{gathered}
$$

(iv) Differential or integrodifferential equations:

$$
\begin{aligned}
& {\left[\frac{B_{n}}{n !} D_{x}^{n}+\cdots+\frac{B_{j+1}}{(j+1) !} D_{x}^{j+1}+\left(\frac{B_{j}}{j !}-j y\right) D_{x}^{j}\right.} \\
& \left.+\frac{B_{j-1}}{(j-1) !} D_{x}^{j-1}+\cdots+\left(\frac{1}{2}-x\right) D_{x}+n\right] B_{n}^{(j)}(x, y)=0, \\
& {\left[\left(x-\frac{1}{2}\right) D_{y}+j D_{x}^{-(j-1)^{2}} D_{y}^{j-1}+j y D_{x}^{-(j-1)^{2}} D_{y}^{j}\right.} \\
& \left.\quad-\sum_{k=1}^{n-1} \frac{B_{n-k+1}}{(n-k+1) !} D_{x}^{-(j-1)(n-k)} D_{y}^{n-k+1}-(n+1) D_{x}^{(j-1)}\right] B_{n}^{(j)}(x, y)=0, \\
& {\left[\left(x-\frac{1}{2}\right) D_{x}^{(j-1)(n-1)} D_{y}+(j-1)(n-1) D_{x}^{(j-1)(n-1)-1} D_{y}\right.} \\
& \quad+j D_{x}^{(j-1)(n-j)}\left(D_{y}^{j-1}+y D_{y}^{j}\right)-\sum_{k=1}^{n-1} \frac{B_{n-k+1}}{(n-k+1) !} D_{x}^{(j-1)(k-1)} D_{y}^{n-k+1} \\
& \left.\quad-(n+1) D_{x}^{(j-1) n}\right] B_{n}^{(j)}(x, y)=0, \quad n \geq j .
\end{aligned}
$$

Note that the last equation can easily be derived by differentiating $(j-1)(n-1)$ times with respect to $x$ both sides of the preceding one, and does not contain antiderivatives for $n \geq j$. 


\section{The 2D Appell polynomials $R_{n}^{(j)}(x, y)$}

For any $j \geq 2$, the $2 \mathrm{D}$ Appell polynomials $R_{n}^{(j)}(x, y)$ are defined by means of the generating function

$$
G_{A}^{(j)}(x, y ; t):=A(t) e^{x t+y t^{j}}=\sum_{n=0}^{\infty} R_{n}^{(j)}(x, y) \frac{t^{n}}{n !} .
$$

Even in this general case, the polynomial $R_{n}^{(j)}(x, y)$ is isobaric of weight $n$ so that it does not contain the variable $y$, for every $n=0,1, \ldots, j-1$.

(i) Explicit forms of the polynomials $R_{n}^{(j)}$ in terms of the Hermite-Kampé de Fériet polynomials $H_{n}^{(j)}$ and vice versa:

$$
\begin{aligned}
R_{n}^{(j)}(x, y) & =\sum_{h=0}^{n}\left(\begin{array}{l}
n \\
h
\end{array}\right) \mathscr{R}_{n-h} H_{n}^{(j)}(x, y) \\
& =n ! \sum_{h=0}^{n} \frac{\mathscr{R}_{n-h}}{(n-h) !} \sum_{r=0}^{[h / j]} \frac{x^{h-j r} y^{r}}{(h-j r) ! r !},
\end{aligned}
$$

where the $\mathscr{R}_{k}$ are the Appell numbers appearing in the definition (2.5);

$$
H_{n}^{(j)}(x, y)=\sum_{k=0}^{n}\left(\begin{array}{l}
n \\
k
\end{array}\right) Q_{n-k} R_{k}^{(j)}(x, y),
$$

where the $Q_{k}$ are the coefficients of the Taylor expansion in a neighborhood of the origin of the reciprocal function $1 / A(t)$.

(ii) Recurrence relation: it is useful to introduce the coefficients of the Taylor expansion

$$
\frac{A^{\prime}(t)}{A(t)}=\sum_{n=0}^{\infty} \alpha_{n} \frac{t^{n}}{n !}
$$

The following linear homogeneous recurrence relation for the generalized Appell polynomials $R_{n}^{(j)}(x, y)$ holds:

$$
\begin{gathered}
R_{0}^{(j)}(x, y)=1 \\
R_{n}^{(j)}(x, y)=\left(x+\alpha_{0}\right) R_{n-1}^{(j)}(x, y)+\left(\begin{array}{c}
n-1 \\
j-1
\end{array}\right) j y R_{n-j}^{(j)}(x, y) \\
+\sum_{k=0}^{n-2}\left(\begin{array}{c}
n-1 \\
k
\end{array}\right) \alpha_{n-k-1} R_{k}^{(j)}(x, y) .
\end{gathered}
$$


(iii) Shift operators:

$$
\begin{gathered}
L_{n}^{-}:=\frac{1}{n} D_{x}, \quad L_{n}^{+}:=\left(x+\alpha_{0}\right)+\frac{j}{(j-1) !} y D_{x}^{j-1}+\sum_{k=0}^{n-1} \frac{\alpha_{n-k}}{(n-k) !} D_{x}^{n-k}, \\
\mathscr{L}_{n}^{-}:=\frac{1}{n} D_{x}^{-(j-1)} D_{y}, \\
\mathscr{L}_{n}^{+}:=\left(x+\alpha_{0}\right)+\frac{j}{(j-1) !} y D_{x}^{-(j-1)^{2}} D_{y}^{j-1}+\sum_{k=0}^{n-1} \frac{\alpha_{n-k}}{(n-k) !} D_{x}^{-(j-1)(n-k)} D_{y}^{n-k} .
\end{gathered}
$$

(iv) Differential or integrodifferential equations:

$$
\begin{aligned}
& {\left[\frac{\alpha_{n-1}}{(n-1) !} D_{x}^{n}+\cdots+\frac{\alpha_{j}}{j !} D_{x}^{j+1}+\left(\frac{\alpha_{j-1}+j y}{(j-1) !}\right) D_{x}^{j}\right.} \\
& \left.+\frac{\alpha_{j-2}}{(j-2) !} D_{x}^{j-1}+\cdots+\left(x+\alpha_{0}\right) D_{x}-n\right] R_{n}^{(j)}(x, y)=0, \\
& {\left[\left(x+\alpha_{0}\right) D_{y}+\frac{j}{(j-1) !} D_{x}^{-(j-1)^{2}}\left(y D_{y}^{j}+D_{y}^{j-1}\right)\right.} \\
& \left.+\sum_{k=1}^{n-1} \frac{\alpha_{n-k}}{(n-k) !} D_{x}^{-(j-1)(n-k)} D_{y}^{n-k+1}-(n+1) D_{x}^{j-1}\right] R_{n}^{(j)}(x, y)=0, \\
& {\left[\left(x+\alpha_{0}\right) D_{x}^{(j-1)(n-1)} D_{y}+(j-1)(n-1) D_{x}^{(j-1)(n-1)-1} D_{y}\right.} \\
& +\frac{j}{(j-1) !} D_{x}^{(j-1)(n-j)}\left(y D_{y}^{j}+D_{y}^{j-1}\right)+\sum_{k=1}^{n-1} \frac{\alpha_{n-k}}{(n-k) !} D_{x}^{(j-1)(k-1)} D_{y}^{n-k+1} \\
& \left.\quad-(n+1) D_{x}^{n(j-1)}\right] R_{n}^{(j)}(x, y)=0, \quad n \geq j .
\end{aligned}
$$

\section{References}

[1] R. P. Agarwal, A propos d'une note de M. Pierre Humbert, C. R. Acad. Sci. Paris 236 (1953), 2031-2032 (French).

[2] P. Appell, Sur une classe de polynômes, Ann. Sci. Ecole Norm. Sup. (2) 9 (1880), 119-144 (French).

[3] P. Appell and J. Kampé de Fériet, Fonctions Hypergéométriques et Hypersphériques. Polynômes d'Hermite, Gauthier-Villar, Paris, 1926.

[4] G. Bretti, M. X. He, and P. E. Ricci, On quadrature rules associated with Appell polynomials, Int. J. Appl. Math. 11 (2002), no. 1, 1-14.

[5] G. Bretti and P. E. Ricci, Multidimensional extensions of the Bernoulli and Appell polynomials, to appear in Taiwanese J. Math.

[6] Euler polynomials and the related quadrature rule, Georgian Math. J. 8 (2001), no. 3, 447-453.

[7] G. Dattoli, S. Lorenzutta, and C. Cesarano, Finite sums and generalized forms of Bernoulli polynomials, Rend. Mat. Appl. (7) 19 (1999), no. 3, 385-391. 
[8] G. Dattoli, P. E. Ricci, and C. Cesarano, Differential equations for Appell type polynomials, Fract. Calc. Appl. Anal. 5 (2002), no. 1, 69-75.

[9] G. Dattoli, P. E. Ricci, and H. M. Srivastava, Two-index multidimensional Gegenbauer polynomials and their integral representations, Math. Comput. Modelling 37 (2003), no. 3-4, 283-291.

[10] K. Douak, The relation of the d-orthogonal polynomials to the Appell polynomials, J. Comput. Appl. Math. 70 (1996), no. 2, 279-295.

[11] A. Erdélyi, W. Magnus, F. Oberhettinger, and F. G. Tricomi, Higher Transcendental Functions. Vols. I, II, McGraw-Hill, New York, 1953.

[12] C. Frappier, Representation formulas for entire functions of exponential type and generalized Bernoulli polynomials, J. Austral. Math. Soc. Ser. A 64 (1998), no. 3, 307-316.

[13] H. W. Gould and A. T. Hopper, Operational formulas connected with two generalizations of Hermite polynomials, Duke Math. J. 29 (1962), 51-63.

[14] M. X. He and P. E. Ricci, Differential equations of some classes of special functions via the factorization method, to appear in J. Comput. Appl. Math.

[15] - Differential equation of Appell polynomials via the factorization method, J. Comput. Appl. Math. 139 (2002), no. 2, 231-237.

[16] L. Infeld and T. E. Hull, The factorization method, Rev. Mod. Phys. 23 (1951), no. 1, 21-68.

[17] M. E. H. Ismail, Remarks on "Differential equation of Appell polynomials via the factorization method", J. Comput. Appl. Math. 154 (2003), no. 1, 243-245.

[18] P. Natalini and A. Bernardini, A generalization of the Bernoulli polynomials, J. Appl. Math. (2003), no. 3, 155-163.

[19] B. Nath, A generalization of Bernoulli numbers and polynomials, Ganita 19 (1968), no. 1, 9-12.

[20] I. M. Sheffer, A differential equation for Appell polynomials, Bull. Amer. Math. Soc. 41 (1935), 914-923.

[21] H. M. Srivastava and H. L. Manocha, A Treatise on Generating Functions, Wiley, New York, 1984.

[22] J. Stoer, Introduzione all'Analisi Numerica, Zanichelli, Bologna, 1972.

[23] F. G. Tricomi and A. Erdélyi, The asymptotic expansion of a ratio of gamma functions, Pacific J. Math. 1 (1951), 133-142.

[24] D. V. Widder, The Heat Equation, Academic Press [Harcourt Brace Jovanovich Publishers], New York, 1975.

Gabriella Bretti: Dipartimento di Metodi e Modelli Matematici per le Scienze Applicate, Università degli Studi di Roma "La Sapienza," 00161 Roma, Italy

E-mail address: bretti@dmmm.uniroma1.it

Pierpaolo Natalini: Dipartimento di Matematica, Università degli Studi Roma Tre, 00146 Roma, Italy

E-mail address: natalini@mat.uniroma3.it

Paolo E. Ricci: Dipartimento di Matematica, Istituto "Guido Castelnuovo", Università degli Studi di Roma "La Sapienza," 00185 Roma, Italy

E-mail address: riccip@uniroma1.it 


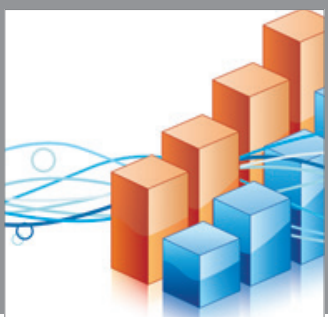

Advances in

Operations Research

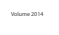

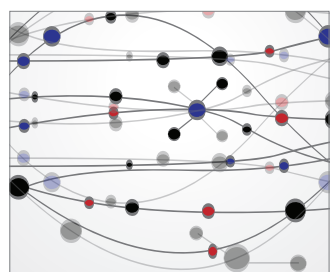

\section{The Scientific} World Journal
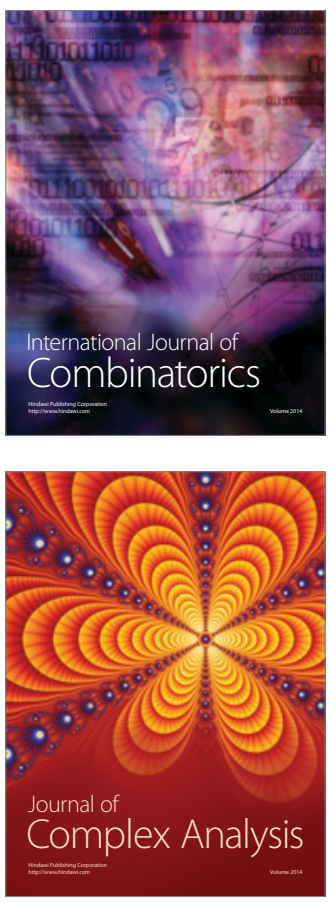

International Journal of

Mathematics and

Mathematical

Sciences
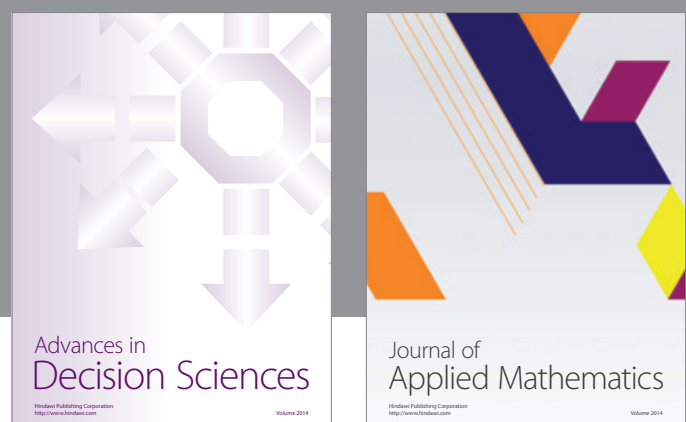

Journal of

Applied Mathematics
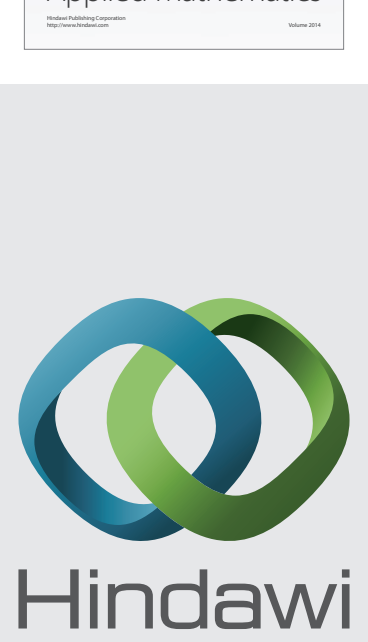

Submit your manuscripts at http://www.hindawi.com
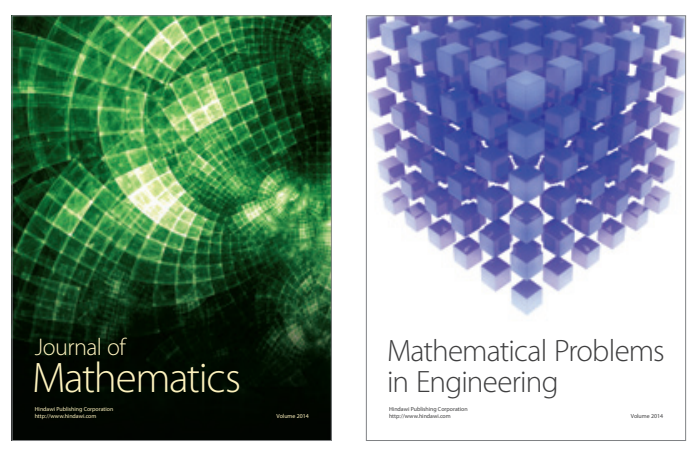

Mathematical Problems in Engineering
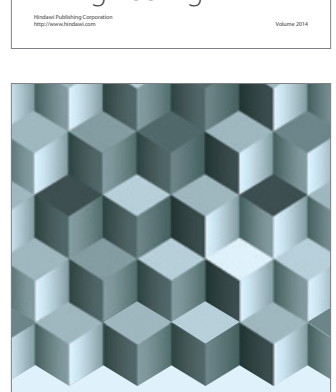

Journal of

Function Spaces
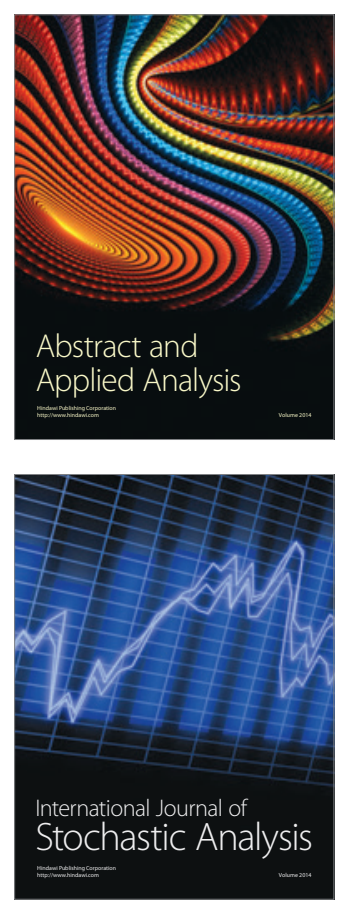

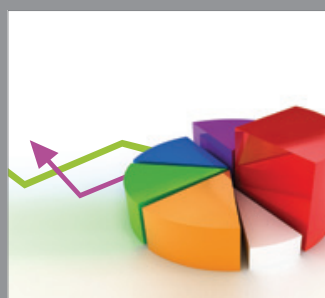

ournal of

Probability and Statistics

Promensencen
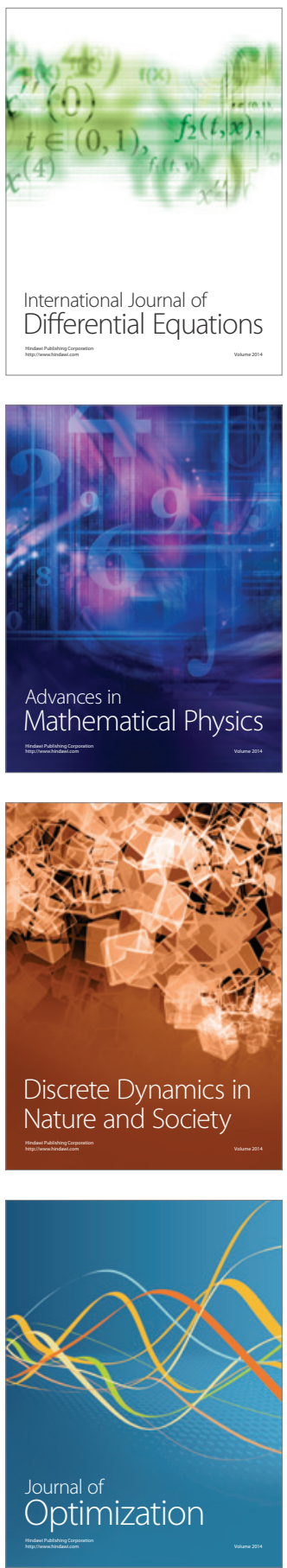